\title{
Sciatic nerve grafting and inoculation of FGF-2 promotes improvement of motor behavior and fiber regrowth in rats with spinal cord transection
}

\author{
Fausto Pierdoná Guzen*, Joacil Germano Soares, Leandro Moura de Freitas, \\ José Rodolfo Lopes de Paiva Cavalcanti, Francisco Gilberto Oliveira, John Fontenele Araújo, \\ Jeferson de Souza Cavalcante, Judney Cley Cavalcante, Expedito Silva do Nascimento Jr and \\ Miriam Stela Maris de Oliveira Costa \\ Laboratory of Neuroanatomy, Department of Morphology, Bioscience Center, Federal University of Rio Grande \\ do Norte, Natal-RN, Brazil
}

\begin{abstract}
Purpose: Failure of severed adult central nervous system (CNS) axons to regenerate could be attributed with a reduced intrinsic growing capacity. Severe spinal cord injury is frequently associated with a permanent loss of function because the surviving neurons are impaired to regrow their fibers and to reestablish functional contacts. Peripheral nerves are known as good substrate for bridging CNS trauma with neurotrophic factor addition. We evaluated whether fibroblastic growth factor 2 (FGF-2) placed in a gap promoted by complete transection of the spinal cord may increase the ability of sciatic nerve graft to enhance motor recovery and fibers regrow.

Methods: We used a complete spinal cord transection model. Rats received a $4 \mathrm{~mm}$-long gap at low thoracic level and were repaired with saline (control) or fragment of the sciatic nerve (Nerve) or FGF-2 was added to nerve fragment (Nerve+FGF-2) to the grafts immediately after complete transection. The hind limbs performance was evaluated weekly for 8 weeks by using motor behavior score (BBB) and sensorimotor tests-linked to the combined behavior score (CBS), which indicate the degree of the motor improvement and the percentage of functional deficit, respectively. Neuronal plasticity were evaluated at the epicenter of the injury using MAP-2 and GAP-43 expression.

Results: Spinal cord treatment with sciatic nerve and sciatic nerve plus FGF-2 allowed recovery of hind limb movements compared to control, manifested by significantly higher behavioral scores. Higher amounts of MAP-2 and GAP-43 immunoreactive fibers were found in the epicenter of the graft when FGF-2 was added.

Conclusions: FGF-2 added to the nerve graft favored the motor recovery and fiber regrowth. Thus, these results encourage us to explore autologous transplantation as a novel and promising cell therapy for treatment of spinal cord lesion.
\end{abstract}

Keywords: Neuroregeneration, sciatic nerve graft, spinal cord, fibroblastic growth factor-2 and motor behavior

${ }^{*}$ Corresponding author: Fausto Pierdoná Guzen, M.D., Full Professor, Department of Biomedic Sciences, Health Sciences Faculty, State University of Rio Grande do Norte, 59607-360, Mossoró, Rio Grande do Norte, Brazil. Tel.: +55 84 33152248; E-mail: faustoguzen@uern.br.
Abbreviations
CNS
FGF-2
PNG 


$\begin{array}{ll}\text { SCs } & \text { Schwann Cells } \\ \text { SCI } & \text { Spinal Cord Injury }\end{array}$

\section{Introduction}

One important approach used in attempts to regain functional recovery after a spinal cord injury (SCI) has included transplantation strategies that are designed to provide a favorable environment for regrowth $\mathrm{Xu}$ et al., 1995; Cheng et al., 1996; Ramon-Cueto et al., 2000; Kubasak et al., 2008). A favorable environment for axon regeneration after a complete spinal cord transection can be created by a combination of a peripheral nerve graft (PNG) transplantation and neurotrophic factors. We have reported that this combinatory treatment in adult complete spinal rats results in significant improvement in motor behavior (Guzen et al., 2009).

The family of fibroblastic growth factors (FGFs) encompasses, to date, more than 20 distinct polypeptides of mesodermal origin that play pivotal roles in several biological processes, such as embryonic development (Gospodarowicz et al., 1987; Thisse and Thisse, 2005), angiogenesis (Presta et al., 2005), and tumorigenesis (Grose and Dickson, 2005). Several studies have found that multiple FGFs, but predominantly FGF-1 and FGF-2, are expressed in the central nervous system (CNS) and are key regulators of CNS development and functions (Hattori et al., 1997a; 1997b; Yamashita et al., 2000; Dono, 2003).

Study shows that FGF-1 and FGF-2 are mainly located in the Schwann cells (SCs), FGF-1 was primarily localized in the cytoplasm while FGF-2 was predominantly localized in the nucleus (Kayton and Aktas, 2000). SCs exhibited increased FGF immunoreactivity with increased time in culture (Neuberger and De Vries, 1993).

Evidence of FGF-1 and FGF-2 mitogenic and neurotrophic activities includes their ability to enhance the survival and outgrowth of various neuronal cell types, such as neocortical, hippocampal, cerebellar, dopaminergic, spinal cord, and sensory neurons isolated from adult CNS (Matsuda et al., 1990; Peulve et al., 1994; Himmelseher et al., 1997; Dono, 2003).

The ability of FGFs to act as potent neurotrophic factors has been extensively demonstrated. FGF-2 prevents axotomy-induced death of glutamatergic neurons (Peterson et al., 1996). Administration of FGF-2 in vivo improves rat sensorimotor functions, reduces focal ischemia-induced infarct size ( $\mathrm{Li}$ and Stephenson, 2002), stimulates functional recovery of rats subjected to motor cortex lesion (Monfils et al., 2005).

The effects of the high molecular weight FGF-2 isoforms $(21,000 \mathrm{~mol}$. wt, 23,000 mol. wt) were investigated on dissociated rat mesencephalic dopaminergic neurons (Grothe et al., 2000). Recombinant FGF-2 isoforms were administered on embryonic dopaminergic neurons in vitro, and Schwann cells over-expressing the high molecular weight isoforms were co-cultured with dopaminergic neurons. This was the first demonstration that the high molecular weight isoforms mediate a neurotrophic activity. Exogenous high molecular weight FGF-2 isoforms stimulated the survival of mesencephalic dopaminergic neurons and protected them from neurotoxicity by 6-hydroxydopamine. In addition, co-culture of dopaminergic neurons with high molecular weight FGF-2 over-expressing SC revealed an increased survival and neurite formation of the mesencephalic dopaminergic neurons, even compared with exogenous administration of FGF-2 isoforms (Grothe et al., 2000).

For other systems, reports strongly suggest that FGF-2 is involved in locomotor recovery after SCI. Indeed, in adult rats, a significant and rapid upregulation of FGF-2 expression was observed both above and below the epicenter of a compression injury (Follesa et al., 1994; Mocchetti et al., 1996; Zai et al., 2005). The up-regulation in the distal spinal cord is thought to play a prominent role in motoneuron survival below the injury and in partial recovery of locomotor function over time (Teng et al., 1998). Furthermore, FGF-2 administration following a moderate or a severe SCI promotes hindlimb movement recovery in the adult rat (Rabchevsky et al., 1999; 2000).

The aim of this study was to analyze whether FGF-2 added to sciatic nerve fragments grafted in a $4 \mathrm{~mm}$ long gap of double complete transected spinal cord of adult rats may potentiate the motor behavior and tissue recovery.

\section{Materials and methods}

\subsection{Animal treatment}

Pathogen free adult male Wistar rats $(n=30)$ from Federal University of Pernambuco, Pernambuco, Brazil (body weight [b.w.] 180-200 g), were used in 
the present study. The rats were taken from a longestablished colony that has been maintained using the San Poiley outbreeding method (Poiley, 1960). Rats were kept under controlled temperature with free access to food pellets and tap water. The study was conducted according to protocols approved by the Animal Care and Use Ethic Committee at the Federal University of Rio Grande do Norte and in accordance with the Guide for the Care and Use of Laboratory Animals adopted by the National Institutes of Health.

\subsection{Microneurosurgery}

Rats were pre-anesthetized with Ketamine Chloridrate $10 \% \quad 0.1 \mathrm{ml} / 100 \mathrm{gr}$ and Xylazine $2 \%$ $0.01 \mathrm{ml} / 100 \mathrm{gr}$ (Agener União, Brasil) and then anesthetized with isoflurane inhalation (Isoforine ${ }^{\circledR}$ ) (Cristália, Brasil). Briefly, a small laminectomy at the tenth/eleventh thoracic levels was performed; the dura mater was incised longitudinally and pulled laterally. Complete transections created a 4 mm-long gap on the eleventh/twelfth spinal cord levels. A gelfoam soaked in $10 \mu \mathrm{l}$ of saline was left at the bottom of the gap close to the vertebral body in 10 rats, other group of 10 rats received gelfoam filled with sciatic nerve fragment, additionally 10 rats received sciatic nerve fragment and $10 \mu \mathrm{l}$ of a FGF-2 solution ( $1 \mu \mathrm{g} / 10 \mu \mathrm{l} / \mathrm{animal}$; Sigma, USA) diluted in PBS. We have not considered further concentrations because higher doses may be toxic to neurons. Two mm-long sciatic nerve fragments were grafted carefully close to the edges of the spinal cord stumps, thus maintained in a position rostral and adjacent to the filled gelfoam. Muscular layers and skin were then sutured. All animals received sodium cephalexin (Eurofarma, Brasil, $40 \mathrm{mg} / \mathrm{kg} /$ day b.w, im.) prior to surgery. The animals were allowed to recover from anesthesia and were returned to their home cages. Sciatic nerve fragments were obtained from a separate group of adult Wistar rats, as described previously (Casella et al., 1996), just before the spinal cord injury procedures. Fragments were removed from the rat sciatic nerves, bilaterally, trimmed out in small pieces of $4 \mathrm{~mm}$-long. Moreover, fragments were washed several times and maintained in a $100 \mathrm{~mm}$ dish with Leibovitz's 15 medium (L-15, Gibco) at room temperature for about 30 minutes while cord injuries have been performed in the host rats.

\subsection{Behavioral analysis}

\subsection{1. $B B B$}

Hindlimb performance was evaluated using the open field locomotor test (Basso et al., 1995), which consists in the evaluation of rat hindlimb patterns of recovery after spinal cord lesions. The scores varied from 0 to 21 and were divided in three blocks: from 0 to 7 corresponded to the initial recovery of the movement in the hindlimb junctions, from 8 to 13 , steps and coordination were evaluated and finally from 14 to 21 fine movements performed during locomotion were tested. Two observers, unaware of the experimental procedures, performed the test twice a week for two months after surgery until the day of euthanize.

\subsubsection{Combined behavior score (CBS)}

A series of sensorimotor tests that compose the CBS has been described elsewhere (Gale et al., 1985). Briefly: Motor score. To assess hindlimb deficit during locomotion, a modified version of the scale described by Tarlov was used. The animal was observed for spontaneous activity in an open field. Each hindlimb was evaluated individually. Toe spread. Spreading of the toes was observed, a reflex elicited as the rat was picked up by its body and the legs allowed hanging free. Placing. The rat was supported at the trunk and the legs allowed hanging free. As the dorsal and lateral aspects of each foot were rubbed against a hard edge, the reflex to place the foot on top of the edge was analyzed. Withdrawal reflexes in response to: (a) Extension. The reflex to withdraw the hindlimb by flexion of the leg when extended was observed as the rat was held down on a table by the body and the hindlimb pulled in a caudal extension. (b) Pain. The withdrawal reflex to pain was elicited by holding the animal still on a bench top and very briefly pinching the pad of the hind foot pad with a pair of Backhaus towel clamps. The stimulus applied does not have sufficient strength or duration to cause injury. (c) Pressure. Withdrawal to pressure was assessed as the animal was held and the hind foot squeezed between thumb and forefinger. Righting. The rat was placed on a table, held by the lower half of the trunk, and then rolled over on its back. Inclined Plane. The rats were individually placed head down on an adjustable inclined plane covered by a rubber mat constructed according to the apparatus described by Rivlin and Tator (Gale et al., 1985). The angle of the plane was gradually increased from $0^{\circ}$ until the rat could not maintain its position for 5 seconds. To 
avoid the effects of motion on the animal, the inclined plane was held stationary at the point that the rat lost its position. The rat was placed on the mat again, and the angle was adjusted as described above. Final readings to the nearest $5^{\circ}$ were taken from the stationary position. Normal uninjured rats remained on the plane to an angle of 50 to $60^{\circ}$ inclination. Moreover, CBS represents the percentage of impairment of sensorimotor function. The CBS tests were evaluated weekly for two months. Moreover, the data of each CBS test were pooled monthly.

\subsubsection{Tissue preparation, sectioning and samppling}

Animals were deeply anaesthetized with Isoforine ${ }^{\circledR}$ (Cristália, Brasil) and euthanized 2 months after surgery by a transcardiac perfusion with $100 \mathrm{ml}$ saline $0.9 \%$ at room temperature followed by $500 \mathrm{ml}$ of fixation fluid $\left(4^{\circ} \mathrm{C}\right)$ over a period of 6 minutes as previous descriptions (DeLeon et al., 1994; do Carmo Cunha et al., 2007). The fixative consisted of $4 \%$ paraformaldehyde (w/v) in $0.1 \mathrm{M}$ phosphate buffer, $\mathrm{pH}$ 7.4. The spinal cord epicenter was removed, kept in the fixative solution at $4^{\circ} \mathrm{C}$ for 90 minutes, and then rinsed in 10\% sucrose (Merck, Germany) dissolved in $0.1 \mathrm{M}$ phosphate buffered saline (PBS), $\mathrm{pH} 7.4$, for 48 hours. The adjacent DRG was also removed and stored at $-70^{\circ} \mathrm{C}$ until use. Serial $20 \mu \mathrm{m}$ thick transversal frozen sections were obtained from the spinal cord with a microtome (Leica, SM 2000R, Germany). The sections were carefully evaluated for visualization of the spinal cord. Every 10th section was sampled in series and was submitted to immunolabelling.

\subsubsection{Immunohistochemical procedures for $M A P-2$ and GAP-43}

Immunoreactivity was developed by the avidinbiotin peroxidase technique (Cintra et al. , 1994; Fuxe et al. , 1994). Series of sections were washed $2 \times 10$ minutes in $0.1 \mathrm{M} \mathrm{PBS}, \mathrm{pH} 7.4$, at room temperature and incubated with 5\% normal goat serum (NGS, Sigma) for 30 minutes at room temperature. Series were then incubated for 24 hours at $4{ }^{\circ} \mathrm{C}$ with one of the following antisera: mouse monoclonal antisera against microtubule associated protein 2 (MAP-2) (Abcam, diluted $1: 2000)$ and growth associated protein 43 (GAP-43) (Abcam, diluted $1: 2600$ ). The antibodies were diluted in PBS containing 0.3\% Triton X-100 (Sigma) and $1 \%$ bovine serum albumin (Sigma). The series of sections were washed again in PBS $(2 \times 10$ minutes $)$ and incubated with biotinylated either goat anti-mouse and anti-rabbit immunoglobulins diluted $1: 1000$ (Jackson Labs, USA) for 1 hour, depending on the host species in which the primary antiserum was raised. The sections were washed again in PBS and incubated with an avidin-biotin peroxidase complex (both diluted $1: 100$, Vectastain, Vector, for 90 minutes). Immunoreactivity was visualized using 3-3'-diaminobenzidine tetrahydrocloride (DAB, Sigma) as a chromogen and $\mathrm{H}_{2} \mathrm{O}_{2}$ $(0.05 \%, v / v$, Sigma) for 4 minutes.

\subsubsection{Morphometric/microdensitometric image analysis}

The MAP-2 and GAP-43 immunoreactivity was measured by count of fibers in one section per rat in the central regions and obtained the means per group. An optical microscope (Olympus BX41) in bright field was used. The counts and measurements were made using the software Image J. Digital images of representative sections were obtained using a digital video camera (Nikon DXM1200).

\subsection{Statistical analysis}

Statistical analyses were performed using the analysis of variance (ANOVA) and significant interactions were followed-up by Tukey and Bonferoni post-test comparison. All statistical analyses were performed using SPSS 18 , and significance was set at $P<0.05$.

\section{Results}

Positive effects $(p<0.0001)$ on the time-dependent progression of the BBB and CBS scores were seen in the lesioned rats treated with sciatic nerve graft in the presence or absence of FGF-2 compared with lesioned rats that received only saline (Fig. 1). It should be noticed that the degree of amelioration in the BBB and CBS parameters were small, since $\mathrm{BBB}$ values did not reach 6 point in the scale (Fig. 1A) and CBS score was about $26 \%$ by the end of the two month post surgery (Fig. 1B).

In the analysis of the BBB was observed an improvement of motor-treated groups in the sciatic nerve with or without addition of FGF-2 compared to saline group in the first week of the period of analysis $p<0.05$. From the second to the eighth week the groups nerve and nerve+FGF-2 showed an improved motor response compared to saline control $(p<0.001)$. However, the 

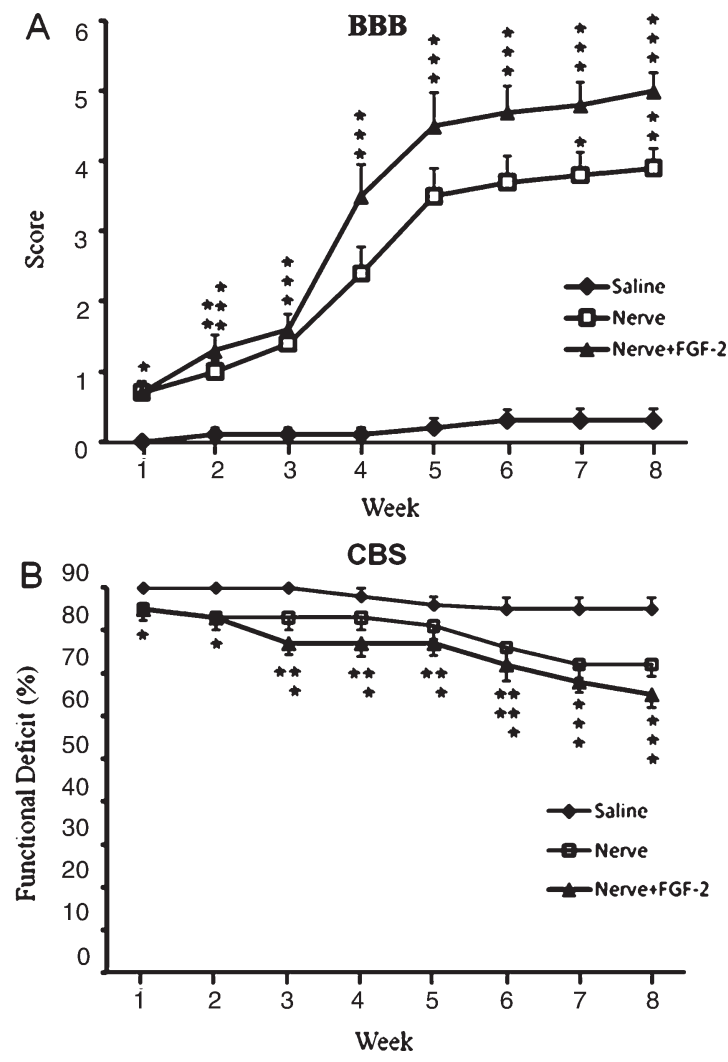

Fig. 1. Time-course of BBB (A) and CBS (combining behavior score, B) levels of rats that received a 4 mm-long gap promoted by a complete double spinal cord transection at a low thoracic level saline administrates or grafted with a sciatic nerve fragment with local addition (NERVE+FGF-2 $\star$ right) or not (NERVE-FGF-2 $\star$ left) of fibroblastic growth factor 2 . The analyses were conducted weekly for two months after surgery. CBS levels indicated the percent of impaired sensorimotor functions, thus the time-dependent decreases of the parameter indicated an amelioration of functional impairments. Furthermore, increases of the BBB scores indicated the gain of motor function. Means \pm S.E.M. ${ }^{\star} p<0.05,{ }^{\star}{ }^{\star} p<0.01$, $\star \star \star p<0.001$, according to ANOVA - Tukey and Bonferoni.

last two weeks (seventh week $p<0.05$; eighth week $p<0.001$ ) the group nerve+FGF-2 enhanced the motor response when compared to the nerve, demonstrating an additional effect of FGF-2. In the final analysis, the saline group did not reach a 1 point, the nerve group was approximately 4 points, and group nerve+FGF-2 approximately 6 points in the BBB scale (Fig. 1A).

In the analysis of CBS (Fig. 1B) the motor parameters in animals that received implant of nerve and nerve+FGF-2 were also improved compared to saline group. However, no motor improvement was statistically significant between groups nerve and nerve+FGF-2 as observed in the BBB test.
The data of several functional tests employed in the evaluation of motor recovery in the spinal cord lesioned rats showed higher degree of performance in the animals of the nerve and nerve+FGF-2 groups in all the two months when compared to saline. The Tarlov motor behavior was improved in the third and seventh week of the period of analysis involving the nerve $p<0.05$ and nerve+FGF-2 groups $p<0.001$. In six $p<0.05$ and eight $p<0.001$ weeks it was detected significant improvement of motor parameters of nerve+FGF-2 group compared to saline. In one, two, four and five weeks no improvement in motor response of the treated groups was seen compared to control (Fig. 2A).

In conducting the tests and Toe Spread Placing no motor response of the saline group behavior was detected. Motor response was more pronounced in the nerve+FGF-2 group when compared to nerve group $p<0.0001$ (Fig. 2B and C).

In tests Withdrawal reflexes to extension, pain and pressure behavioral motor responses were present in the final period of analysis in the saline group. However, in the nerve groups with or without addition of FGF-2 resulted in improved behavioral response in the first weeks perpetuating until the end of the analysis. The behavioral responses were more pronounced in nerve+FGF-2 group in relation to the nerve group (Fig. 2D-F). Considering Withdrawal reflexes pain, only the nerve+FGF-2 group showed significant behavioral response in the fourth and fifth weeks $p<0.05$ compared to saline group (Fig. 2E). In righting reflex test, the parameters of the motor nerve and nerve+FGF-2 groups were also enhanced compared to the saline $p<0.0001$ (Fig. 2G).

Response also appears in the inclined plane test where the nerve+FGF-2 group showed relevant response in the first and last weeks $p<0.05$ compared to saline (Fig. 2H).

Coronal sections from the epicenter of the injury subjected to immunohistochemistry for MAP-2 exhibited immunoreactivity to this protein in the group treated with sciatic nerve graft (Fig. 3A and B), being better viewed in the group treated with sciatic nerve graft plus FGF-2 (Fig. 3C and D). No MAP-2-immunoreactive fibers were visualized in sections of the saline group (not shown). The sciatic nerve plus FGF-2 group showed a value of 260.1 grains for MAP-2-immunoreactive fibers against to 248.2 from nerve group $\left.{ }^{\star} p=0.004\right)$. Animals treated with sciatic nerve grafts added or not FGF-2 showed 

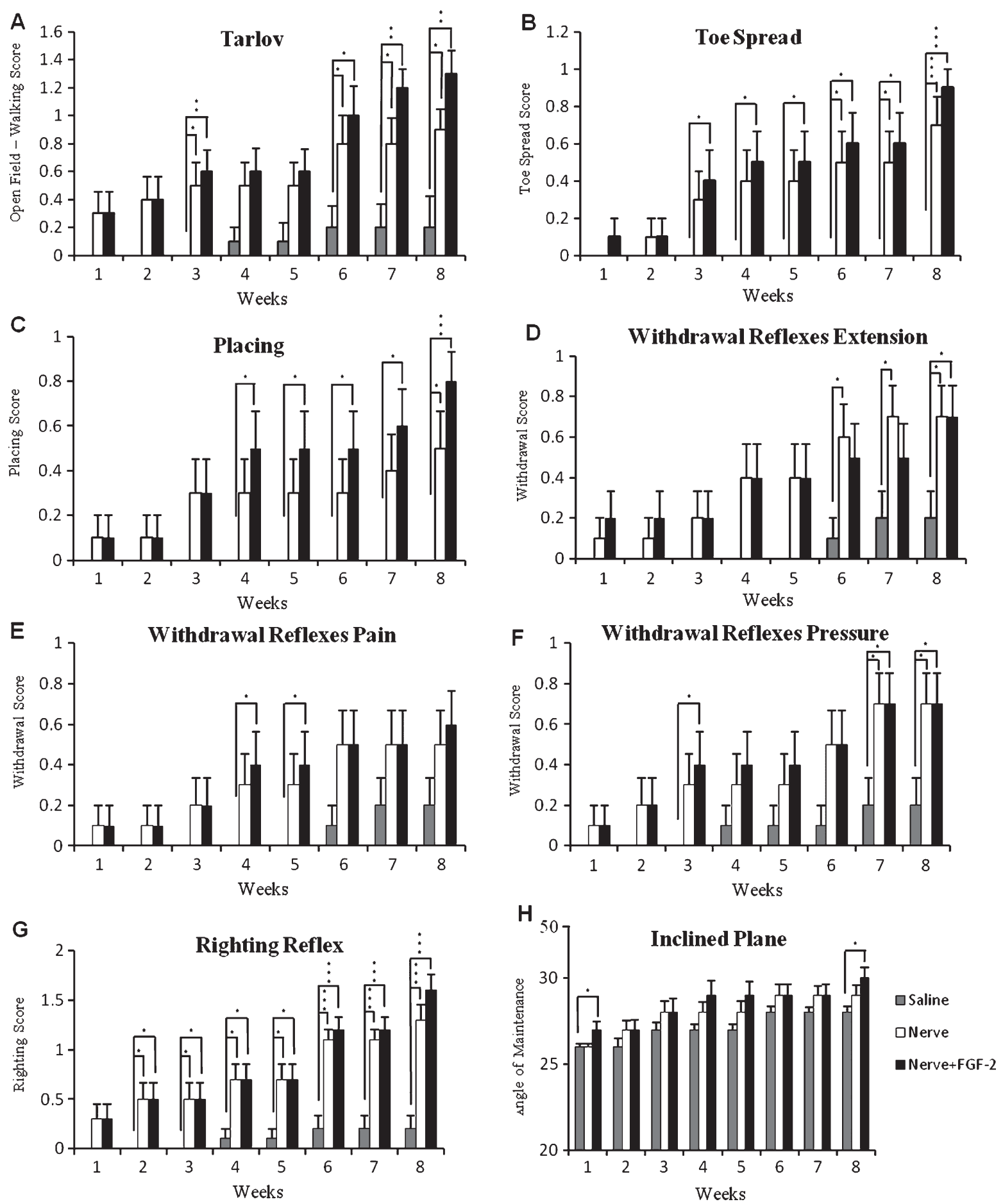

Fig. 2. Time-course of functional analyses of rats after a $4 \mathrm{~mm}$-long gap promoted by a complete double spinal cord transection at a low thoracic level that were treated with grafts of sciatic nerve fragment with (NERVE+FGF-2) or without fibroblastic growth factor (NERVE-FGF-2). The following tests were applied: Tarlov (A), Toe Spread (B), Placing (C), Withdrawal reflex to extension (D), Withdrawal reflex to pain (E),Withdrawal reflex to pressure (F), Righting reflex $(\mathrm{G})$ and Incline plane $(\mathrm{H})$. The analyses were conducted weekly. See text for details. Means \pm S.E.M. ${ }^{\star} p<0.05,{ }^{\star}{ }^{\star} p<0.01,{ }^{\star}{ }^{\star}{ }^{\star} p<0.001$ according to ANOVA - Tukey and Bonferoni. 

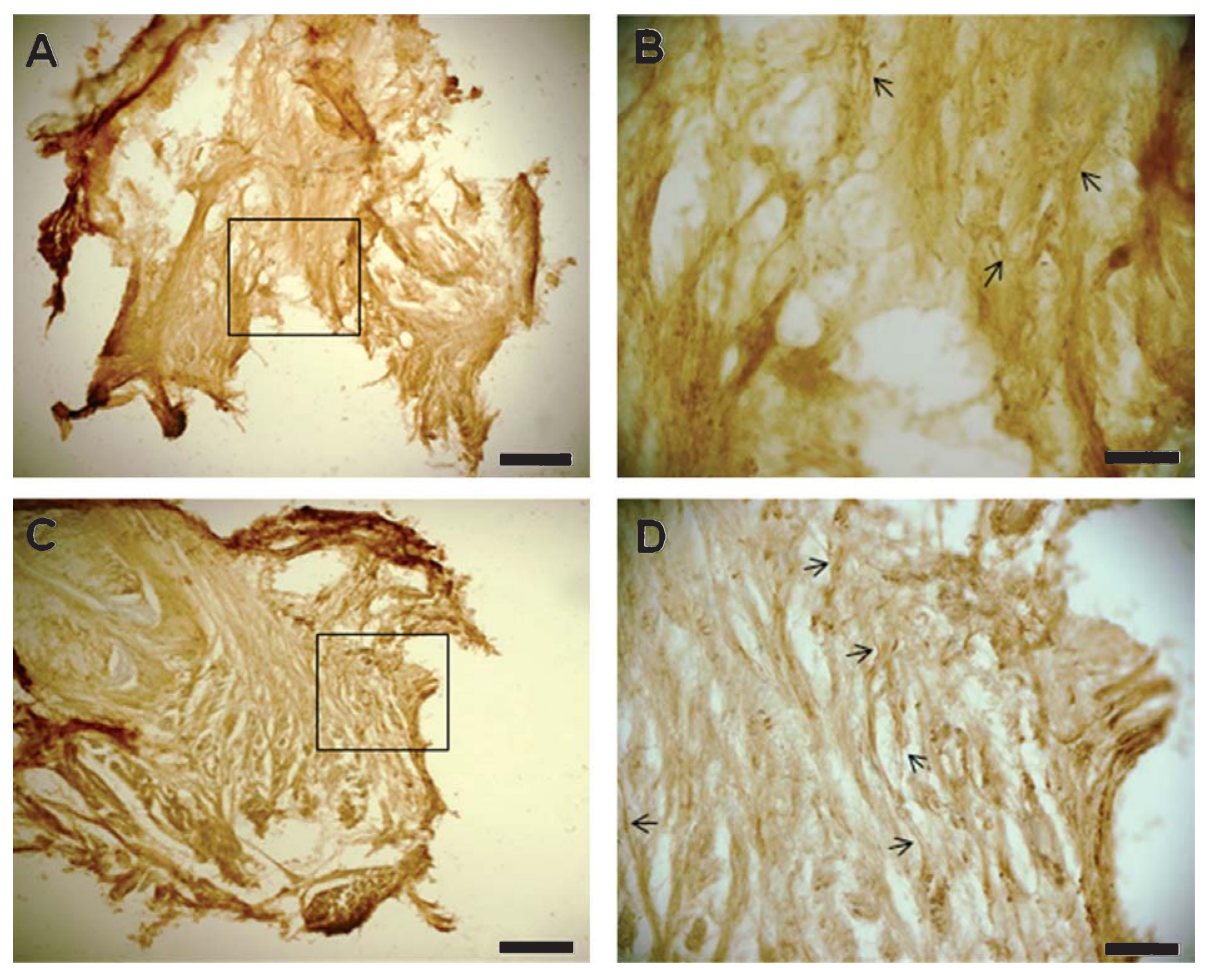

Fig. 3. Photomicrographs of coronal sections at the epicenter of injury to the animal that received complete spinal cord transaction treated with sciatic nerve graft (A and B) and sciatic nerve graft added FGF-2 (C and D), immunoreactive for MAP-2. Illustrations show fibers at the epicenter of the injury at the epicenter of the lesion (arrows). Bars: $200 \mu \mathrm{m}$ (A and C) and $50 \mu \mathrm{m}$ (B and D).

significant effect $(\star \star \star \star p=0.0001)$ when compared to saline group (Fig. 4).

GAP-43 immunohistochemistry on sections from the epicenter of the injury, revealed the presence of this protein in both sciatic nerve graft (Fig. 5A and B) and sciatic nerve graft plus FGF-2 (Fig. 5C and D) groups, although better visualized in the last one. No GAP-43-immunoreactive fibers were visualized in sections of the saline group (not shown). Quantitatively, the group that received sciatic nerve grafted added FGF-2 exhibited 294 fibers over 277 for the sciatic nerve graft group ( $\left.{ }^{\star} p=0.008\right)$. The groups treated with sciatic nerve grafts added or not FGF-2 showed significant effect $(\star \star \star \star x<0.0001)$ when compared to saline group (Fig. 6).

\section{Discussion}

When axons in the adult mammalian CNS are injured they undergo regenerative sprouting, but these sprouts fail to grow for more than a few millimeters through CNS tissue. It is clear that environmental factors play a role in restricting axonal growth within a mature CNS environment, because mature neurons can extend axons long distances within a peripheral nerve graft but terminate shortly after reentering a CNS environment (Aguayo et al. , 1979; 1981; Richardson et al. , 1980; 1984; David and Aguayo, 1981; 1985). Moreover, axotomized central mature neurons are able to respond to a variety of external influences that affect their survival and axonal elongation. For a successful regeneration, the regenerative fibers must trespass all adverse obstacles at wound, which may be impaired with tissue discontinuity (Reier et al. , 2002).

Fibers might also be able to growth throughout pathways in order to encounter correct targets for innervations (Bray et al. , 1987; Ramer et al. , 2000). We employed here a model of a complete axotomy of the rat spinal cord in order to analyze the effects of the manipulation of the wound repair zone, by interposing a sciatic nerve graft, on motor behavior recovery.

Addition of exogenous neurotrophic factors potentiates axonal growth by chronically injured neurons 


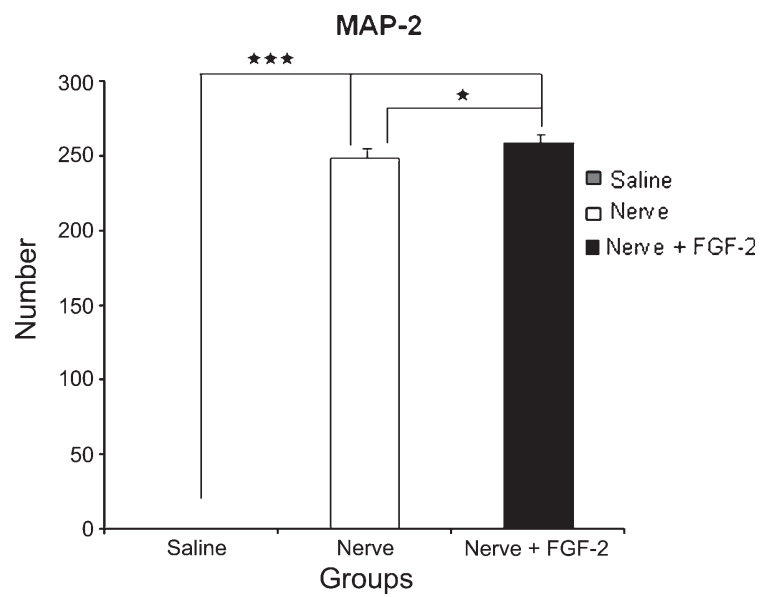

Fig. 4. MAP-2-immunoreactivity profiles at the epicenter of the lesion in spinal cord of animals treated with saline, sciatic nerve graft (Nerve) or sciatic nerve graft added FGF-2 (Nerve+FGF-2), after complete transection of the spinal cord. Means \pm S.E.M. ${ }^{\star} p<0.05$,

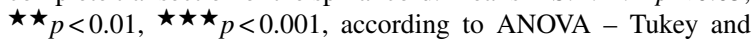
Bonferroni. if scar tissue is excised (Ye and Houle, 1997). We have analyzed whether the presence of FGF-2 in the nerve graft might potentiate the motor outcome and regenerative responses. Results showed that the nerve graft repair in the presence of FGF-2 constitutes an important experimental advance to favor functional recovery of rats. Furthermore, several reports have indicated spontaneous reorganization of tissue in the injury site that includes Schwann cell invasion, slow formation of astroglial scarring and sprouting of descending axons (Brook et al. , 1998; Hill et al. , 2004).

Data indicated that the distal spinal cord stump, the target for the regenerated descending fibers, also responded by short and long term up-regulation of FGF-2 mRNA expression. This up-regulation could facilitate the reestablishment of the functional innervation of spinal locomotor networks below the injury by regenerated brainstem and intraspinal descending neurons. In line with this assumption, the expression
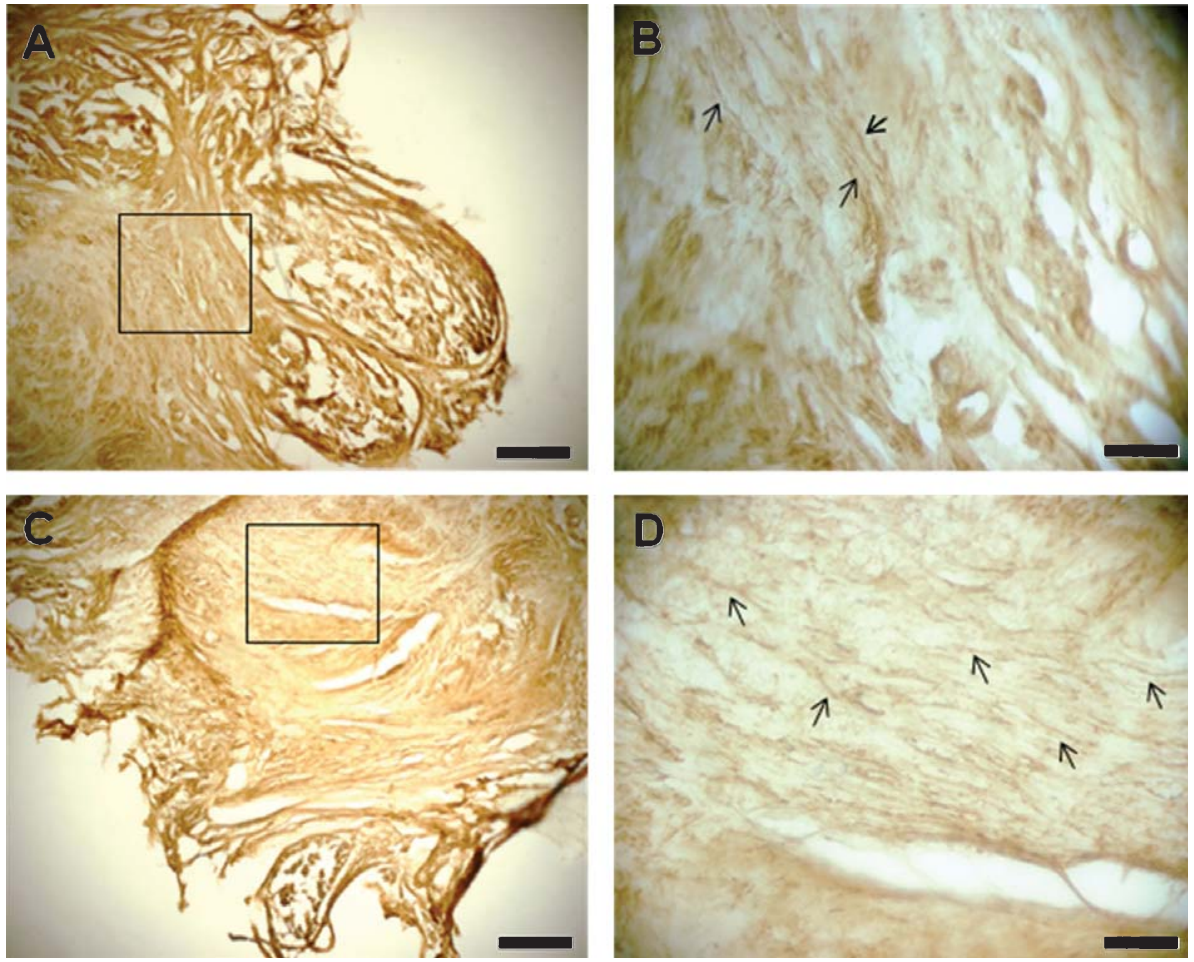

Fig. 5. Photomicrographs of coronal sections at the epicenter of injury to the animal that received complete spinal cord transaction treated with sciatic nerve graft (A and B) and sciatic nerve graft added FGF-2 (C and D), immunoreactive for GAP-43. Illustrations show fibers at the epicenter of the injury at the epicenter of the lesion (arrows). Bars: $200 \mu \mathrm{m}$ (A and C) and $50 \mu \mathrm{m}$ (B and D). 


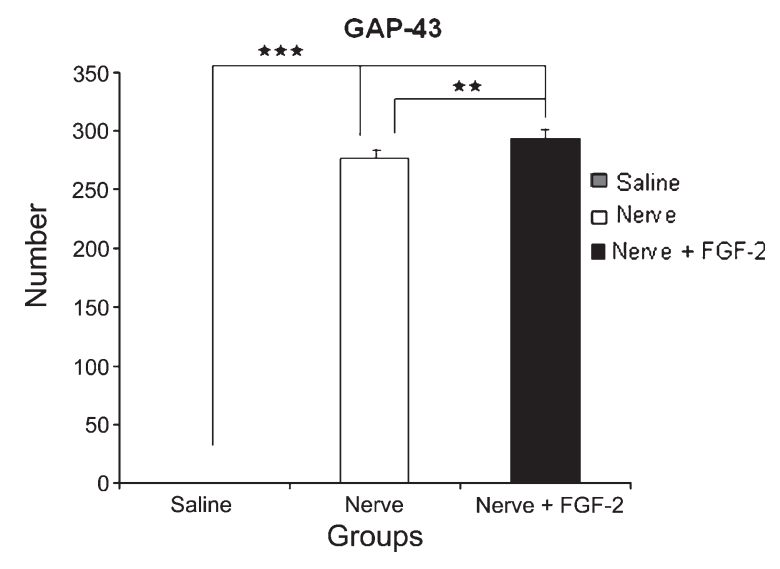

Fig. 6. Distribution of GAP-43 immunoreactivity at the epicenter of spinal cord injury of animals treated with saline, sciatic nerve graft (Nerve) and sciatic nerve graft added FGF-2 (Nerve+FGF-2). Means \pm S.E.M. ${ }^{\star} p<0.05,{ }^{\star}{ }^{\star} p<0.01,{ }^{\star}{ }^{\star}{ }^{\star} p<0.001$, according to ANOVA - Tukey and Bonferroni.

of FGF-2 mRNA and protein was also shown to be upregulated in the distal cord after a compression injury or a complete spinal cord transection in infant rats, but not in adult rats (Follesa et al., 1994; Qi et al. , 2003). This differential expression of FGF-2 has been related to the higher regenerative capacity of some descending tracts and functional recovery in spinally transected infant rats (Wakabayashi et al., 2001).

Kawamata et al., (1997) reported an increase in growth-associated protein 43 (GAP-43) immunoreactivity, a molecular marker of axonal sprouting, in the intact sensorimotor cortex contralateral to cerebral infarcts (induced by middle cerebral artery occlusion) following FGF-2 treatment, suggesting that FGF-2 enhances functional improvement after stroke via stimulation of neuronal sprouting in the intact brain. FGF-2 also significantly increases sprouting by the cholinergic septo dentate pathway following entorhinal cortex injury (Ramirez et al. , 1999), and astrocyte-associated fibronectin (Mahler et al. , 1997), an axonal growthpromoting molecule (Liesi et al. , 1986; Matthiessen et al. , 1989).

In vivo application of FGF-2 during the phase of ontogenetic neuron death, however, did not confirm this notion (Oppenheim et al. , 1992). Moreover, in vitro studies showed that exogenous application of FGF-2 induced brainstem neurite outgrowth (Moftah, 2008). The temporal course of FGF-2 mRNA expression observed in other study could be related to the period of progressive recovery of hindlimb locomo- tion in spinally transected animals (Chevallier et al. , 2004).

Intrathecal infusion of exogenous FGF-2 following severe contusion SCI in rats significantly enhances functional recovery of hindlimb movements in the absence of significant tissue sparing at the injury site (Rabchevsky et al. , 2000). This phenomenon suggests that impulses for standing from supraspinal centers do not reach motoneurons innervating the muscles of the hind limbs during that period.

\section{Conclusion}

The addition of exogenous FGF-2 to a sciatic nerve fragment grafted in a gap of the rat spinal cord submitted to complete transections was able to improve motor behavior recovery. The results emphasized that the manipulation of the microenvironment at the wound might amplify the regenerative capacity of central neurons.

\section{Acknowledgments}

This study was supported by finding from the National Counsel of Technological and Scientific Development (CNPq) and the Coordination for Improvement of High Level Staff (CAPES).

\section{References}

Aguayo, A., David, S., Richardson, P. \& Bray, G. (1979). Axonal elongation in peripheral and central nervous system transplants. Adv cell Neurobiol, 3, 215-234.

Aguayo, A.J., David, S. \& Bray, G.M. (1981). Influences of the glial environment on the elongation of axons after injury: Transplantation studies in adult rodents. J Exp Biol, 95, 231-240.

Basso, D.M., Beattie, M.S. \& Bresnahan, J.C. (1995). A sensitive and reliable locomotor rating scale for open field testing in rats. J Neurotrauma, 12, 1-21.

Bray, G.M., Villegas-Perez, M.P., Vidal-Sanz, M. \& Aguayo, A.J. (1987). The use of peripheral nerve grafts to enhance neuronal survival, promote growth and permit terminal reconnections in the central nervous system of adult rats. J Exp Biol, 132, 5-19.

Brook, G.A., Schmitt, A.B., Nacimiento, W., Weis, J., Schroder, J.M. \& Noth, J. (1998). Distribution of B-50(GAP-43) mRNA and protein in the normal adult human spinal cord. Acta Neuropathol, 95, 378-386. 
Casella, G.T., Bunge, R.P. \& Wood, P.M. (1996). Improved method for harvesting human Schwann cells from mature peripheral nerve and expansion in vitro. Glia, 17, 327-338.

Cheng, H., Cao, Y. \& Olson, L. (1996). Spinal cord repair in adult paraplegic rats: Partial restoration of hind limb function. Science, 273, 510-513

Chevallier, S., Landry, M., Nagy, F. \& Cabelguen, J.M. (2004). Recovery of bimodal locomotion in the spinal-transected salamander, Pleurodeles waltlii. Eur J Neurosci, 20, 1995-2007.

David, S. \& Aguayo, A.J. (1981). Axonal elongation into peripheral nervous system "bridges" after central nervous system injury in adult rats. Science, 214, 931-933.

David, S. \& Aguayo, A.J. (1985). Axonal regeneration after crush injury of rat central nervous system fibres innervating peripheral nerve grafts. J Neurocytol, 14, 1-12.

Dono, R. (2003). Fibroblast growth factors as regulators of central nervous system development and function. Am J Physiol Regul Integr Comp Physiol, 284, R867-R881.

Follesa, P., Wrathall, J.R. \& Mocchetti, I. (1994). Increased basic fibroblast growth factor mRNA following contusive spinal cord injury. Brain Res Mol Brain Res, 22, 1-8.

Gale, K., Kerasidis, H. \& Wrathall, J.R. (1985). Spinal cord contusion in the rat: Behavioral analysis of functional neurologic impairment. Exp Neurol, 88, 123-134.

Gospodarowicz, D., Neufeld, G. \& Schweigerer, L. (1987). Fibroblast growth factor: Structural and biological properties. J Cell Physiol Suppl, 5, 15-26.

Grose, R. \& Dickson, C. (2005). Fibroblast growth factor signaling in tumorigenesis. Cytokine Growth Factor Rev, 16, 179-186.

Grothe, C., Schulze, A., Semkova, I., Muller-Ostermeyer, F., Rege, A. \& Wewetzer, K. (2000). The high molecular weight fibroblast growth factor-2 isoforms (21,000 mol. wt and 23,000 mol. wt) mediate neurotrophic activity on rat embryonic mesencephalic dopaminergic neurons in vitro. Neuroscience, 100, 73-86.

Guzen, F.P., de Almeida Leme, R.J., de Andrade, M.S., de Luca, B.A. \& Chadi, G. (2009). Glial cell line-derived neurotrophic factor added to a sciatic nerve fragment grafted in a spinal cord gap ameliorates motor impairments in rats and increases local axonal growth. Restor Neurol Neurosci, 27, 1-16.

Hattori, Y., Miyake, A., Mikami, T., Ohta, M. \& Itoh, N. (1997a). Transient expression of FGF-5 mRNA in the rat cerebellar cortex during post-natal development. Brain Res Mol Brain Res, 47, 262-266.

Hattori, Y., Yamasaki, M., Konishi, M. \& Itoh, N. (1997b). Spatially restricted expression of fibroblast growth factor-10 mRNA in the rat brain. Brain Res Mol Brain Res, 47, 139-146.

Hill, A.E., Gardner, I.A., Carpenter, T.E. \& Stover, S.M. (2004). Effects of injury to the suspensory apparatus, exercise, and horseshoe characteristics on the risk of lateral condylar fracture and suspensory apparatus failure in forelimbs of thoroughbred racehorses. Am J Vet Res, 65, 1508-1517.

Himmelseher, S., Pfenninger, E. \& Georgieff, M. (1997). Effects of basic fibroblast growth factor on hippocampal neurons after axonal injury. J Trauma, 42, 659-664.

Kawamata, T., Dietrich, W.D., Schallert, T., Gotts, J.E., Cocke, R.R., Benowitz, L.I. \& Finklestein, S.P. (1997). Intracisternal basic fibroblast growth factor enhances functional recovery and upregulates the expression of a molecular marker of neuronal sprouting following focal cerebral infarction. Proc Natl Acad Sci U S A, 94, 8179-8184.

Kayton, R.J. \& Aktas, R.G. (2000). Electron microscopic immunolocalization of basic fibroblast growth factor in peripheral nerves. Histochem Cell Biol, 114, 413-419.

Kubasak, M.D., Jindrich, D.L., Zhong, H., Takeoka, A., McFarland, K.C., Munoz-Quiles, C., Roy, R.R., Edgerton, V.R., RamonCueto, A. \& Phelps, P.E. (2008). OEG implantation and step training enhance hindlimb-stepping ability in adult spinal transected rats. Brain, 131, 264-276.

Li, Q. \& Stephenson, D. (2002). Postischemic administration of basic fibroblast growth factor improves sensorimotor function and reduces infarct size following permanent focal cerebral ischemia in the rat. Exp Neurol, 177, 531-537.

Liesi, P., Kirkwood, T. \& Vaheri, A. (1986). Fibronectin is expressed by astrocytes cultured from embryonic and early postnatal ratbrain. Exp Cell Res, 163, 175-185.

Mahler, M., Ferhat, L., BenAri, Y. \& Represa, A. (1997). Effects of tenascin-C in cultured hippocampal astrocytes: NCAM and fibronectin immunoreactivity changes. Glia, 20, 231-242.

Matsuda, S., Saito, H. \& Nishiyama, N. (1990). Effect of basic fibroblast growth factor on neurons cultured from various regions of postnatal rat brain. Brain Res, 520, 310-316.

Matthiessen, H.P., Schmalenbach, C. \& Muller, H.W. (1989). Astroglia-released neurite growth-inducing activity for embryonic hippocampal-neurons is associated with laminin bound in a sulfated complex and free fibronectin. Glia, 2, 177-188.

Mocchetti, I., Rabin, S.J., Colangelo, A.M., Whittemore, S.R. \& Wrathall, J.R. (1996). Increased basic fibroblast growth factor expression following contusive spinal cord injury. Exp Neurol, 141, 154-164.

Moftah, M., Landry, M., Nagy, F. \& Cabelguen, J.M. (2008). Fibroblast growth factor- 2 mRNA expression in the brainstem and spinal cord of normal and chronic spinally transected urodeles. J Neurosci Res, 86, 3348-3358.

Monfils, M.H., Driscoll, I., Vandenberg, P.M., Thomas, N.J., Danka, D., Kleim, J.A. \& Kolb, B. (2005). Basic fibroblast growth factor stimulates functional recovery after neonatal lesions of motor cortex in rats. Neuroscience, 134, 1-8.

Neuberger, T.J. \& De Vries, G.H. (1993). Distribution of fibroblast growth factor in cultured dorsal root ganglion neurons and Schwann cells. II. Redistribution after neural injury. J Neurocytol, 22, 449-460.

Oppenheim, R.W., Prevette, D. \& Fuller, F. (1992). The lack of effect of basic and acidic fibroblast growth-factors on the naturallyoccurring death of neurons in the chick-embryo. J Neurosci, $12,2726-2734$.

Peterson, D.A., Lucidi-Phillipi, C.A., Murphy, D.P., Ray, J. \& Gage, F.H. (1996). Fibroblast growth factor-2 protects entorhinal layer II glutamatergic neurons from axotomy-induced death. $\mathrm{J} \mathrm{Neu}$ rosci, 16, 886-898.

Peulve, P., Laquerriere, A., Hemet, J. \& Tadie, M. (1994). Comparative effect of alpha-MSH and b-FGF on neurite extension of fetal rat spinal cord neurons in culture. Brain Res, 654, 319-323. 
Poiley, S.M. (1960). In: Proceedings of Animal Care Panel. 10, 159166.

Presta, M., Dell'Era, P., Mitola, S., Moroni, E., Ronca, R. \& Rusnati, M. (2005). Fibroblast growth factor/fibroblast growth factor receptor system in angiogenesis. Cytokine Growth Factor Rev, 16, 159-178.

Qi, M.L., Wakabayashi, Y., Haro, H. \& Shinomiya, K. (2003). Changes in FGF-2 expression in the distal spinal cord stump after complete cord transection: A comparison between infant and adult rats. Spine (Phila Pa 1976), 28, 1934-1940.

Rabchevsky, A.G., Fugaccia, I., Fletcher-Turner, A., Blades, D.A., Mattson, M.P. \& Scheff, S.W. (1999). Basic fibroblast growth factor (bFGF) enhances tissue sparing and functional recovery following moderate spinal cord injury. J Neurotrauma, 16, 817830 .

Rabchevsky, A.G., Fugaccia, I., Turner, A.F., Blades, D.A., Mattson, M.P. \& Scheff, S.W. (2000). Basic fibroblast growth factor (bFGF) enhances functional recovery following severe spinal cord injury to the rat. Exp Neurol, 164, 280-291.

Ramer, M.S., Priestley, J.V. \& McMahon, S.B. (2000). Functional regeneration of sensory axons into the adult spinal cord. Nature, $403,312-316$

Ramirez, J.J., Finklestein, S.P., Keller, J., Abrams, W., George, M.N. \& Parakh, T. (1999). Basic fibroblast growth factor enhances axonal sprouting after cortical injury in rats. Neuroreport, 10 , 1201-1204.

Ramon-Cueto, A., Cordero, M.I., Santos-Benito, F.F. \& Avila, J. (2000). Functional recovery of paraplegic rats and motor axon regeneration in their spinal cords by olfactory ensheathing glia. Neuron, 25, 425-435.

Reier, P.J., Golder, F.J., Bolser, D.C., Hubscher, C., Johnson, R., Schrimsher, G.W. \& Velardo, M.J. (2002). Gray matter repair in the cervical spinal cord. Prog Brain Res, 137, 49-70.
Richardson, P.M., Mcguinness, U.M. \& Aguayo, A.J. (1980). Axons from Cns neurons regenerate into Pns grafts. Nature, 284, 264265

Richardson, P.M., Issa, V.M.K. \& Aguayo, A.J. (1984). Regeneration of long spinal axons in the rat. Journal of Neurocytology, 13 , 165-182.

Teng, Y.D., Mocchetti, I. \& Wrathall, J.R. (1998). Basic and acidic fibroblast growth factors protect spinal motor neurones in vivo after experimental spinal cord injury. Eur J Neurosci, 10, 798802.

Thisse, B. \& Thisse, C. (2005). Functions and regulations of fibroblast growth factor signaling during embryonic development. Dev Biol, 287, 390-402.

Wakabayashi, Y., Komori, H., Kawa-Uchi, T., Mochida, K., Takahashi, M., Qi, M.L., Otake, K. \& Shinomiya, K. (2001). Functional recovery and regeneration of descending tracts in rats after spinal cord transection in infancy. Spine, 26, 12151222.

Xu, X.M., Guenard, V., Kleitman, N., Aebischer, P. \& Bunge, M.B. (1995). A combination of BDNF and NT-3 promotes supraspinal axonal regeneration into Schwann cell grafts in adult rat thoracic spinal cord. Exp Neurol, 134, 261-272.

Yamashita, T., Yoshioka, M. \& Itoh, N. (2000). Identification of a novel fibroblast growth factor, FGF-23, preferentially expressed in the ventrolateral thalamic nucleus of the brain. Biochem Biophys Res Commun, 277, 494-498.

Ye, J.H. \& Houle, J.D. (1997). Treatment of the chronically injured spinal cord with neurotrophic factors can promote axonal regeneration from supraspinal neurons. Exp Neurol, 143, 70-81.

Zai, L.J., Yoo, S. \& Wrathall, J.R. (2005). Increased growth factor expression and cell proliferation after contusive spinal cord injury. Brain Res, 1052, 147-155. 